\title{
Factors influencing reproductive choices of HIV positive individuals attending primary health care facilities in a South African health district
}

Samuel Agbo ${ }^{1,2,4^{*}}$ (D) and Laetitia C. Rispel ${ }^{3}$

\begin{abstract}
Background: There is global recognition of the reproductive health rights of people living with HIV (PLHIV). The aim of this research study was to explore the reproductive choices, and the factors influencing these choices, of HIV positive patients attending primary health care (PHC) facilities in the Ekurhuleni health district of the Gauteng Province of South Africa.
\end{abstract}

Methods: During 2013, a cross-sectional survey was conducted in the Ekurhuleni health district. At each of three randomly selected community health centres, a random sample of HIV positive patients was selected. After informed consent was obtained, trained fieldworkers administered a structured questionnaire that elicited information on socio-demographics, reproductive choices and knowledge of reproductive options. Survey data were analysed using STATA 13.

Results: The majority of survey participants $(n=430)$ were female $(70 \%)$ and unemployed $(57 \%)$. The mean age of participants was 36.4 years (SD 8.6): 40.8 years (SD 8.7) for men and 34.5 years (SD7.8) for women.

Among survey participants, 46\% expressed a desire for children (95\% Cl: 41.4-50.9). In the multiple logistic regression analysis, predictors of desire for children were age less than 49 years, marriage or living together, and no biological children. The odds of wanting children was 16.48 [95\% Cl: 5.94-45.74] times higher for PLHIV without children, compared with those with two or more children, while for those less than 25 years, the odds of wanting children was 0.78 [95\% Cl: 0. 23-2.59] compared with those older than 50 years.

The PLHIV knowledge on the available reproductive options was limited, with the majority relying on the guidance of the health workers.

Conclusion: Health care providers at PHC level should be educated to address the reproductive health needs of PLHIV. These aspects should be reflected in provincial and national health policies.

Keywords: Reproductive choices, HIV positive patients, Desire for children, HIV\&AIDS, South Africa

\section{Background}

The HIV \& AIDS epidemic continues to rank among the top ten priority conditions that contribute to the global disease burden [1], with sub-Saharan Africa accounting for $66 \%$ of the global HIV burden [2]. Although there is new optimism of achieving an AIDS-free generation by

\footnotetext{
*Correspondence: sam2012agbo@gmail.com; Samuel.Agbo@wits.ac.za 1 Department of Family Medicine, Faculty of Health Sciences, University of the Witwatersrand, Johannesburg, South Africa

${ }^{2}$ School of Public Health, Faculty of Health Sciences, University of the Witwatersrand, Johannesburg, South Africa

Full list of author information is available at the end of the article
}

2030 [3], there is also recognition that a rapid scale-up of existing programmes is needed to achieve this ambitious goal $[3,4]$. South Africa continues to be a priority country for intervention [4], with an estimated 6.4 million People Living with HIV (PLHIV) [5-9].

Sexual transmission of HIV infection continues to account for the majority of global HIV infections [10]. The improvement in HIV management especially the introduction of anti-retroviral therapy (ART) has resulted in improved quality of life and increased life expectancy of HIV positive persons [4]. In recent years, 
the sexual and reproductive health rights of PLHIV have been re-iterated, especially the responsibilities of governments to ensure that health care and legal systems support these rights [11]. Consequently, there has been the re-emergence of debates regarding reproductive choices and desires of HIV positive people [12-14]. The WHO has indicated that the unmet need for family planning remains high among HIV positive women, and has emphasised the strengthening of linkages between HIV and sexual and reproductive health programmes [4].

There is a significant body of literature on reproductive choices of and challenges faced by PLHIV [15-25]. Existing evidence suggests that a sizable proportion of PLHIV, regardless of geographical location, have an expressed desire for biological children [17, 21-27]. Factors that influence this desire included younger age, personal health status, having a regular partner, perception that the partner wanted children, knowledge of ART effectiveness, ethnicity, knowledge about contraception or the cultural issues [17, 24, 26, 27]. In sub-Saharan Africa, studies have reported the immense pressure on women to have children regardless of their HIV status [17, 19, 21, 23]. However, another study found that the situation for men was grave as well, with not having children often resulting in stigmatisation and loss of social status among peers [28].

Several studies have found that the attitudes of health care providers influence the reproductive choices of PLHIV [19, 29-35]. Many PLHIV want to discuss sexual and reproductive health needs with their health care providers, but reportedly feel uncomfortable about doing so $[19,29-35]$. The study findings suggest that access to sexual and reproductive health services is critical, while advocacy groups have argued that weak health systems deny PLHIV their sexual and reproductive health and rights [11].

In South Africa, major health sector reforms towards universal health coverage (UHC) underscore the centrality of primary health care (PHC) [36, 37]. It is envisaged that a comprehensive range of health care services, including sexual and reproductive health services, would be delivered at PHC level [37]. In light of the global emphasis on addressing the sexual and reproductive health needs of PLHIV, and the importance of PHC to achieving UHC, the aim of this research study was to explore the reproductive choices, and the factors influencing these choices, of HIV positive patients attending primary health care (PHC) facilities in the Ekurhuleni health district of the Gauteng Province of South Africa.

\section{Methods}

\section{Study setting}

The study was conducted in the Ekurhuleni health district, one of the five health districts in the Gauteng province of South Africa [38, 39].
In 2013, the district had an estimated population of 3 million people and the PHC facilities head count was 5 million with utilisation rate of 2 million, of the public health sector facilities [40, 41]. The Ekurhuleni district health system (DHS) consists of a district hospital, seven community health centres (CHCs) and 87 PHC clinics. These $\mathrm{PHC}$ facilities ( $\mathrm{CHCs}$ and clinics), are staffed primarily by professional nurses, who are supported by generalist medical officers and family physicians. Health care services are provided free of charge i.e. there is no out of pocket payment at these PHC facilities [40].

\section{Ethical considerations}

The study was approved by the University of the Witwatersrand's Human Research Ethics Committee and the provincial health authority. Standard ethical procedures were adhered to, including detailed participant information sheets, obtaining informed consent and ensuring confidentiality of information. A written consent document was signed by all participants who agreed to take part in the research. A distress protocol was developed, and any individuals who needed support and counselling because of emotional distress experienced during data collection were referred to the lay counsellor based at the health facility.

\section{Study population}

The study population consisted of all the HIV positive patients attending public sector PHC facilities within the Ekurhuleni district. In 2013, 143,871 patients were on ART and cared for at these PHC facilities [40]. Each community health centre $(\mathrm{CHC})$ had an average weekly head count of 500 HIV positive or 2000 patients per month, and an average female to male ratio of 70:30 [40, 41].

\section{Study design and sampling}

During 2013, a cross-sectional study was conducted to explore the reproductive choices of HIV positive patients in Ekurhuleni district of Gauteng province, and the factors influencing these choices.

The sampling frame consisted of all HIV positive patients attending the seven $\mathrm{CHCs}$ in Ekurhuleni heath district. The $\mathrm{CHCs}$ were selected as they are well resourced, provide comprehensive $\mathrm{PHC}$ services, have dedicated HIV clinics and attend to a large number of the HIV positive patients.

The required sample size for the study was 369 patients, which was adjusted to 442 to take account of a possible refusal rate of $20 \%$. The eligibility criteria for participation included known HIV-positive patient in the district, 18 to 49 years in the case of women, and 18-60 years in the case of men. These age groups are the bulk of PLHIV in their most reproductive period of life. All patients who 
were too sick to be interviewed or who declined participation were excluded from the study.

One $\mathrm{CHC}$ from each of the two sub-districts - east and south was sampled randomly while the only $\mathrm{CHC}$ from the north was selected. The study participants were selected from these sampled CHCs, using systematic sampling with a random start, until the required sample size was reached.

\section{Data collection}

A pre-tested semi-structured questionnaire, developed in line with the study objectives, and translated into isiZulu and seSotho, the two major local languages, was used to collect data. The questionnaire obtained information on socio-demographic characteristics, HIV treatment, reproductive choices, and knowledge of reproductive options (Additional file 1). Following introduction by a nurse at the selected $\mathrm{CHC}$, the two field workers proceeded with the recruitment processes. These field workers completed secondary school education, and were given both theoretical and practical research field work training.

At each $\mathrm{CHC}$, the questionnaire was administered by these trained field workers (one female and the other male) after informed consent had been obtained. The field workers ensured that each person's privacy was maintained.

\section{Data analysis}

The data were captured in Microsoft excel and analysed using STATA $^{\circ} 13$. Frequency tabulations were done to describe the socio-demographic characteristics of the respondents. In the inferential statistical analysis, the variables examined included the following: associations between socio-demographic characteristics such as: age, marital status, number of children, and level of education, employment and home language, and reproductive desires, reasons for reproductive desires, availability of reproductive services, knowledge of reproductive options, and disclosure of HIV status.

The Chi square test was used to determine the level of association of the factors influencing decision to have a child / children. Univariate logistic regression models were fitted to find factors, which were independently associated with desire to have children. Only factors associated with desire for children that were found to be statistically significant were considered in the model building exercise using a multiple logistic regression model. All statistical tests were carried out at $5 \%$ significance level.

\section{Results}

Socio-demographic characteristics of study participants The majority of study participants (70\%) were women. The mean age of all participants was 36 years (SD 8.6). The mean age of men was 40.8 years compared to
34.5 years for women, and this difference was statistically significant ( $p$-value $<0.05$ ). Marital status also differed significantly by gender with women (49\%) being more likely to be single than men (33\%). The majority of the participants have either completed primary or secondary school ( $44 \%$ and $41 \%$ respectively). There was no significant difference in educational qualification between men and women. The level of employment was $43 \%$ for all participants while Nguni languages were mostly spoken as the home language $(62 \%)$ and followed by Sotho languages (32\%). The mean number of children was 2 (Table 1 ).

\section{HIV diagnosis, disclosure and treatment}

The majority of participants (79.5\%) knew about their HIV status for more than a year, with $9 \%$ finding out about their HIV status in the 6 months preceding the study. The duration since HIV diagnosis was not different between men and women $(p=0.969)$.

The majority of participants indicated that they had disclosed their HIV status (95\%), with 97\% of men and $94 \%$ of women indicating that they had disclosed their HIV status $(p=0.232)$. More women $(71 \%)$ compared to men (54\%) had disclosed their HIV status to family members; while more men (33\%) compared to women $(16 \%)$ disclosed their status to their partner $(p<0.001)$. Only 9\% of study participants reported that they were living openly with HIV, with more men (12\%) in this category.

Most participants (93\%) were on antiretroviral treatment (HAART), although this differed significantly by gender $(p=0.018)$. Men $(97.6 \%)$ were more likely to be on ART compared to women $(91.3 \%)(p=0.018)$. The majority of participants $(77 \%)$ described their current health status as good or excellent. Only $4 \%$ perceived their health to be poor. Perceived differences in health status by gender were not statistically significant.

\section{Reproductive desire and contraception use}

Among study participants, 46\% (95\% CI: 41.4-50.9) expressed a desire to have children in the future, $44 \%$ indicated that they do not wish to have children in the future, while $10 \%$ were unsure. There were no significant differences in reproductive desire by gender ( $p$-value $=0.582$ ), although a slightly higher proportion of men (50\%) compared to women (44\%) expressed the wish to have children in the future (Table 2).

Although 189 of respondents (44\%) indicated that they did not wish to have children in the future, only 71 reported the use of contraceptives.

\section{Reasons for wanting children}

Figure 1 shows the participants' reasons for wanting children. The commonest reasons were wanting more children (36\%) and 'I do not have any child of my own' (22\%). 
Table 1 Socio-demographic characteristics of study participants

\begin{tabular}{|c|c|c|c|c|}
\hline & $\begin{array}{l}\text { Men } \\
(n=128)\end{array}$ & $\begin{array}{l}\text { Women } \\
(n=302)\end{array}$ & $P$-value & $\begin{array}{l}\text { Total } \\
(n=430)\end{array}$ \\
\hline Mean age (SD) & $40.8(8.7)$ & $34.5(7.8)$ & $<0.001$ & $36.4(8.6)$ \\
\hline \multicolumn{5}{|l|}{ Age categories n (\%) } \\
\hline 24 years and below & $2(1.6)$ & $29(9.6)$ & \multirow[t]{4}{*}{$<0.001$} & $31(7.2)$ \\
\hline $25-34$ years & $30(23.4)$ & $125(41.4)$ & & $155(36.1)$ \\
\hline $35-49$ years & $77(60.2)$ & $148(49.0)$ & & $225(52.3)$ \\
\hline 50 years and above & $19(14.8)$ & $0(0)$ & & $19(4.4)$ \\
\hline \multicolumn{5}{|l|}{ Marital status n (\%) } \\
\hline Single & $42(32.8)$ & $148(49.0)$ & \multirow[t]{5}{*}{0.004} & $190(4.2)$ \\
\hline Living together & $35(27.4)$ & $81(26.8)$ & & $116(27.0)$ \\
\hline Married & $38(29.7)$ & $48(15.9)$ & & $86(20.0)$ \\
\hline Divorced & $7(5.5)$ & $9(3.0)$ & & $16(3.7)$ \\
\hline Widowed & $6(4.7)$ & $16(5.3)$ & & $22(5.1)$ \\
\hline \multicolumn{5}{|l|}{ Education } \\
\hline No schooling & $17(12.7)$ & $26(8.6)$ & \multirow[t]{4}{*}{0.375} & $43(9.9)$ \\
\hline Completed primary & $62(46.3)$ & $128(42.5)$ & & $190(43.7)$ \\
\hline Completed secondary & 49 (36.6) & $129(42.9)$ & & $178(40.9)$ \\
\hline Tertiary & $6(4.5)$ & $18(6.0)$ & & $24(5.5)$ \\
\hline Currently Employed & $65(50.8)$ & $120(39.7)$ & 0.034 & $185(43.0)$ \\
\hline \multicolumn{5}{|l|}{ Home Language } \\
\hline Nguni (isiZulu, isiXhosa, isiNdebele, or siSwati) & $80(62.5)$ & $186(61.6)$ & \multirow[t]{3}{*}{0.930} & $266(61.9)$ \\
\hline Sotho (seSotho, Setswana, or isiPedi) & $40(31.3)$ & $98(32.5)$ & & $138(32.1)$ \\
\hline Others & $8(6.3)$ & $18(6.0)$ & & $26(6.0)$ \\
\hline Mean number of own children (SD) & $2.3(1.6)$ & $1.9(1.4)$ & 0.0129 & $2.0(1.4)$ \\
\hline
\end{tabular}

\section{Knowledge of available reproductive methods}

The majority of the participants (55\%) indicated that they will wait for the doctors' advice on the appropriate fertility option and $20 \%$ of the respondents were in favour of the natural method of conception. Participants who expressed a desire for artificial insemination made up $10 \%$ while those who are not certain about the reproductive options available to them were 13\% (Fig. 2).

\section{Factors influencing reproductive desire}

In the multiple logistic regression analysis (Table 3), the main predictors of reproductive desire were: age below 49 years $\{35-49$ years $(\mathrm{AOR}=0.42$; $95 \%$ CI: $0.24-0.72)$, below 25 years $(\mathrm{AOR}=0.78$; 95\% CI: $0.23-2.59)\}$, being

Table 2 Participants' expressed desire for children

\begin{tabular}{|c|c|c|c|c|}
\hline & $\begin{array}{l}\text { Men } \\
(N=128)\end{array}$ & $\begin{array}{l}\text { Women } \\
(N=302)\end{array}$ & $P$-value & $\begin{array}{l}\text { Total } \\
(N=430)\end{array}$ \\
\hline \multicolumn{5}{|c|}{ Wish to have children in future $\mathrm{n}(\%)$} \\
\hline No & $52(41.3)$ & $137(45.5)$ & 0.582 & $189(44.3)$ \\
\hline Yes & $63(50.0)$ & $134(44.5)$ & & $197(46.1)$ \\
\hline Unsure & $11(8.7)$ & $30(10.0)$ & & $41(9.6)$ \\
\hline
\end{tabular}

married $(\mathrm{AOR}=2.21 ; 95 \%$ CI: $1.12-4.35)$ or living together $(\mathrm{AOR}=2.78 ; 95 \% \mathrm{CI}: 1.48-5.21)$ and having no child (AOR $=16.48$; 95\% CI: 5.94-45.74).

\section{Preferred setting for sessions on reproductive health information.}

When asked about the preferred setting respondents would prefer to receive reproductive health information, majority chose seminar or workshop. There were no gender differences observed in the responses $(p<0.388)$. The preferred type of group make-up for sessions on reproductive health had majority (30\%) preferring only men or women's groups compared to individual (23\%) session types.

\section{Discussion}

The majority of the participants were 25-49 years of age, thus supporting existing evidence that HIV primarily affects the economically active section of the population [7, 39, 42, 43]. The findings underscore the importance of addressing the sexual and reproductive health needs of this sub-group of the population. There were high levels of unemployment and a high proportion 


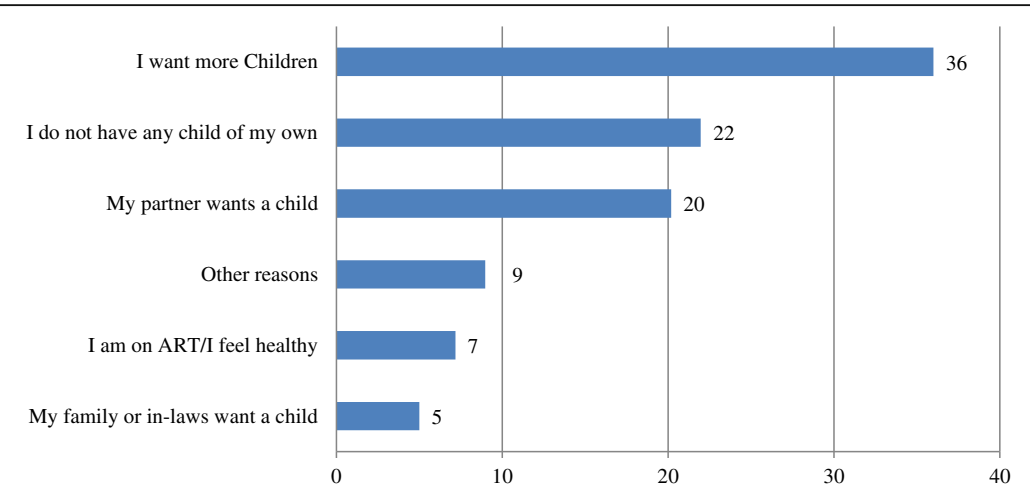

Fig. 1 Reasons for wanting children

of the population without private health insurance (uninsured) which implies dependence on the public health facilities [39, 40, 42, 43]. Hence, the importance of attending to the sexual and reproductive health needs of PLHIV, particularly in the public health sector.

In this study, $50 \%$ of men and $44 \%$ of women expressed a desire to have children. This expressed desire for children is similar to the findings of other studies. Cooper et al. found that in Cape Town, 55\% of female and $43 \%$ of male participants in their study wanted children [19]. Similarly Beyeza-Kashesya et al. [17], found that $64 \%$ of female and $55 \%$ of male HIV positive participants expressed the desire for children. Rispel et al. found that the desire to have children was high amongst HIV-discordant couples interviewed in South Africa and Tanzania [22]. Chen et al. reported similar high rates in their study of fertility desires and intentions of PLHIV in the United States of America [24]. Oladapo et al. likewise reported a high expressed desire for children in their study in Nigeria [44].

Numerous studies have found that the desire for children among Africans is higher, compared to non-Africans
[45-48]. Heard et al., reported that men and women of African ethnicity living in France expressed desire for children was three times higher compared to Europeans [27]. This was attributed to the socio-cultural background of people of African origin: for women, motherhood is considered as a high social status and identity, and as a prestige among peers while for men, on the other hand, fatherhood is critical as it ensures the continuation of the family name and lineage [47-53]. In this study, the population was homogeneous and ethnicity was not a considered variable.

In this study, the multi-variate analysis found that age below 49 years, being married or living together; and having no child were the predictors of reproductive desire (Table 3).

The study found that at age younger than 25 years of age, $80 \%$ of respondents expressed the desire for children, compared to $6 \%$ at age 50 years and above. These research findings are similar to those of other research studies [47-54]. Oladapo et al., found that the reason why younger HIV positive patients had a greater desire for children, was the socio-cultural pressure on young

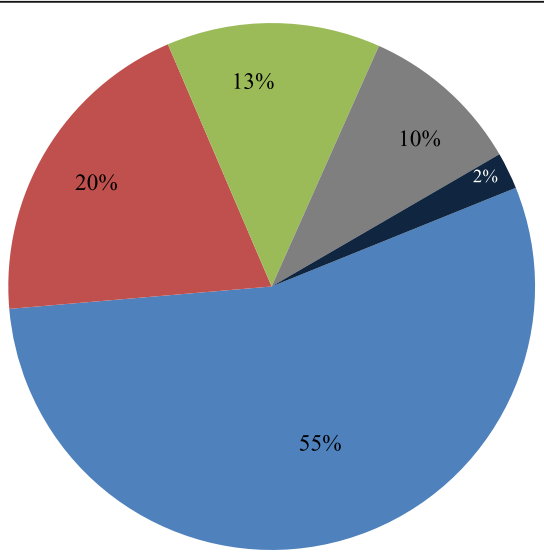

Will wait for the doctor's advice $\quad$ Natural methods $\square$ I don't know $\approx$ Artificial insemination $\square$ Other

Fig. 2 Knowlege of reproductive methods 
Table 3 Multiple logic regression model of factors associated with reproductive desire

\begin{tabular}{|c|c|c|c|c|}
\hline Factor & Level & Unadjusted OR (95\% Cl) & Adjusted OR $(95 \% \mathrm{Cl})$ & $P$-value \\
\hline \multirow[t]{4}{*}{ Age } & Less than 24 years & $2.04(0.72 ; 5.80)$ & $0.78(0.23 ; 2.59)$ & \\
\hline & 25-34 years & 1 (baseline) & 1 (baseline) & 0.0001 \\
\hline & $35-49$ years & $0.42(0.24 ; 0.72)$ & $0.42(0.24 ; 0.72)$ & \\
\hline & 50 years and above & $0.03(0.004 ; 0.25)$ & $0.04(0.004 ; 0.33)$ & \\
\hline \multirow[t]{4}{*}{ Marital Status } & Divorced / Widowed & $0.42(0.19 ; 0.94)$ & $0.75(0.27 ; 2.05)$ & \\
\hline & Married & $0.83(0.49 ; 1.41)$ & $2.21(1.12 ; 4.35)$ & \\
\hline & Living Together & $1.86(1.13 ; 3.07)$ & $2.78(1.48 ; 5.21)$ & \\
\hline & Single & 1 & 1 & 0.0022 \\
\hline \multirow[t]{4}{*}{ No of children } & None & $15.57(5.90 ; 41.10)$ & $16.48(5.94 ; 45.74)$ & \\
\hline & One & $5.65(3.21 ; 9.92)$ & $7.04(3.69 ; 13.42)$ & \\
\hline & $2-3$ & 1 (baseline) & 1 (baseline) & $<0.0001$ \\
\hline & 4 and above & $0.26(0.12 ; 0.58)$ & $0.32(0.14 ; 0.74)$ & \\
\hline \multirow[t]{4}{*}{ Highest Education Level } & Less than primary & 1 (baseline) & - & - \\
\hline & Complete primary & $2.5(1.1 ; 5.5)$ & & \\
\hline & Completed secondary & $3.7(1.7 ; 8.3)$ & & \\
\hline & Tertiary & $3.9(1.3 ; 11.9)$ & & \\
\hline \multirow[t]{2}{*}{ Health Status } & Poor / Okay & 1 (baseline) & - & - \\
\hline & Good / Excellent & $1.6(1.0 ; 2.6)$ & & \\
\hline
\end{tabular}

adults for fulfilment because of the fear of dying early from the disease [44].

Marriage or living together was also found to be one of the predictors of the desire for children. This may imply stability in a relationship and thus increase the desire for wanting children. However, the desire for children decreased among participants with more than one child compared to those with none, so these factors have to be looked at in combination. The inverse relationship between number of children and desire for children was reported by Cooper et al. and Chen et al. $[19,24]$.

A further argument could be advanced that those in marriage or living together might be of advanced age and/or having many children already and consequently may not desire more children. On the other hand, married couples might view the main outcome of marriage as having children and so will desire to have children despite their HIV status. The explanation for the increased desire for children among PLHIV who are either married or living together as independent variable observed in this study cannot be explained easily. Further research may be necessary to determine which of these variables are more strongly correlated to reproductive desires.

This study did not find self-reported health status or being on HAART to be a predictor of reproductive desire. This contrasts with other research findings $[47,55,56]$. In the study by Oladapo et al., ill-health due to advancing HIV infection, evidenced by low CD4 count, was found to be an independent predictor of fertility desire [44]. The socio-cultural fulfilment of child bearing made very sick individuals want to have children before dying [44].

In this study $44 \%$ of participants indicated that they do not want to have children. Among the reasons given by participants for not wanting children were: already had own children, lack of enough resources to care for another child, fear of a baby being born with HIV infection, old age and lack of a stable partner. However, among those not desiring children only $36 \%$ reported contraceptive use. This low usage of contraception was also reported by Smits et al. [44] and Nakayiwa et al. [47].

Most of the respondents who expressed a desire to have children were in favour of the natural method of reproduction (20\%). This could be that the natural method is the only one known by the majority of participants. Although natural reproduction is recommended for PLHIV especially HIV sero-discordant couples on effective HAART regimen, there are specific requirements and guidelines which may not be understood by PLHIV $[19,57]$. Hence, the research participants favouring natural methods may need guidance on the most appropriate method [19].

Thirteen present of respondents did not know the various types of reproductive options available. This lack of knowledge of PLHIV on fertility options may limit their ability to make informed reproductive decisions. It could be due to lack of information on reproductive issues among these PLHIV. The knowledge of available reproductive options by PLHIV has not been a focus of 
most research studies; hence, this finding could not be compared with the findings of other studies.

Most of the respondents (55\%) wanted guidance from the medical personnel on the appropriate reproductive options available to them. This could be due to participants' lack of knowledge about other options such as artificial insemination and assisted reproductive technologies (ART). In several instances the inadequacy on the part of HCWs on the provision of reproductive assistance and their negative attitudes towards PLHIV on sexual and reproductive health made it difficult to meet the needs of their patients $[58,59]$.

The possibility of adoption was not explored in our study but it would be interesting to understand how the situation in Ekurhuleni compares with the negative attitude towards adoption found in a study in Cape Town, South Africa [19].

This was a cross-sectional study carried out in a resource-limited district in Gauteng province, and the findings cannot be generalised to all districts in South Africa, except those with similar features to Ekurhuleni. The respondents in this research were those who attended the public clinics for care and treatment and therefore their responses may differ from those attending private facilities or private general medical practices. The study relied on self-reported information from PLHIV in a health care setting. Hence, there may be social desirability bias, as respondents may have been concerned about possible victimisation or negative consequences for subsequent care at the clinics. However, considerable efforts were made to use trained fieldworkers, and not the staff that provide care at these facilities where the study was conducted. All participants were given clear information and assured of their privacy and confidentiality during and after the interviews.

There are numerous strengths of this research study. This was one of the first studies to examine various aspects of sexual and reproductive health issues of HIV positive patients at a PHC level in Ekurhuleni district. A $100 \%$ response rate was obtained, and collection of data via face-to-face interviews ensured that the views of PLHIV who are not literate were elicited. This study also determined the preferred setting for information sharing and counselling on reproductive desires, an element hitherto unexplored in other studies on SRH and PLHIV.

This study found that there is expressed desire for children among PLHIV and their knowledge on the available reproductive options was limited. However, the participants expressed desire for information on reproductive options was also high. The respondents in this study indicated preference for men or women group discussions on reproductive health information.

There are encouraging developments within South Africa: the national strategic plan (NSP) for HIV/ AIDS and tuberculosis, 2012-2016, overseen by the South African National AIDS Council (SANAC), provides guidance on interventions and activities that will change the incidence and prevalence of HIV, STIs and TB [60]. However, the NSP falls short of guidelines on sexual and reproductive needs of PLHIV [60]. In the short term, sexual and reproductive health service guideline/protocols on reproductive options should be made available to HCWs. All health professionals (doctors and nurses, and other categories) should receive training on the reproductive needs and rights of PLHIV in other to improve their capacity and change their attitudes towards the SRH needs of HIV positive patients [53]. The clinics should also be equipped with educational and demonstration tools and audio-visual materials and equipment to educate PLHIV. In the medium term, the next version of the NSP should include specific goals, objectives, and outcome measures for the sexual and reproductive health needs of PLHIV. This will enable, provincial and district health plans to address the reproductive needs of PLHIV in line with SANAC strategic plan.

\section{Conclusion}

The study generated locally, context specific information in a health district in Gauteng Province on the choices of HIV patients with regards to reproduction and the motivating factors for these choices and as well as their knowledge of fertility options. The study findings suggest that HCPs at primary health care level are critical to ensure that the sexual and reproductive health service needs of PLHIV are met.

\section{Additional file}

Additional file 1: Questionnaire: reproductive choices among HIV positive patients in Ekurhuleni district, Gauteng province. (DOCX 18 kb)

\section{Abbreviations \\ AIDS: Acquired Immune deficiency syndrome; ART: Assisted reproductive technology; ARV: Antiretroviral; CHC: Community health centers; DHS: District health system; HCPs: Health care providers; HCWs: Health care workers; HIV: Human Immunodeficiency Virus; IVF: In-vitro fertilisation; NSP: National strategic plan; PHC: Primary health care; PLHIV: People living with HIV; SANAC: South African National AIDS Council; SRH: Sexual and reproductive health; STI: Sexually transmitted infection; TB: Tuberculosis; UNC: Universal health coverage; WHO: World Health Organisation}

\section{Acknowledgements}

The study was conducted in partial fulfilment of the requirements for the degree of Master of Public Health (MPH) of Samuel Agbo. The participation of the study participants is acknowledged gratefully. We would like to thank the fieldworkers who assisted with data collection. Braimoh Bello and Professor Jonathan Levin are thanked for their assistance with data analysis.

\section{Funding}

This study was funded partially by the WITS Faculty of Health Sciences Research Office. This funding body was not involved in the study design, collection, analysis, and interpretation of the data and in writing the manuscript. 


\section{Availability of data and materials}

The field data collected are stored by the author but not publicly available due to confidentiality concerns.

\section{Authors' contributions}

SA designed the research study, participated in the development of the protocol and questionnaire, supervised the data collection, involved in data capturing, data analysis, and write-up of manuscript. LR supervised SA's MPH research report, participated in design of the study, development of the protocol and questionnaire, provided input on analysis, review of the manuscript drafts and provided editorial assistance. Both authors have read and approved the final version of this manuscript.

\section{Competing interests}

The authors declare that they have no competing interests.

\section{Consent for publication}

Not applicable

\section{Ethics approval and consent to participate}

Ethical clearance for the study was obtained from the University of the Witwatersrand's Human Research Ethics Committee and the Ekurhuleni health district research ethics committees. A written consent document was signed by all participants who agreed to take part in the research.

\section{Publisher's Note}

Springer Nature remains neutral with regard to jurisdictional claims in published maps and institutional affiliations.

\section{Author details}

'Department of Family Medicine, Faculty of Health Sciences, University of the Witwatersrand, Johannesburg, South Africa. ${ }^{2}$ School of Public Health, Faculty of Health Sciences, University of the Witwatersrand, Johannesburg, South Africa. ${ }^{3}$ Centre for Health Policy \& DST/NRF Research Chair, School of Public Health, Faculty of Health Sciences, University of the Witwatersrand, Johannesburg, South Africa. ${ }^{4}$ P.O BOX 12089, Tramshed, Pretoria 0126, South Africa.

Received: 29 September 2016 Accepted: 15 May 2017

Published online: 02 June 2017

\section{References}

1. Murray CJL, Lopez AD. Measuring the Global Burden of Disease. N Engl J Med. 2013:369:448-57.

2. UNAIDS. Fact sheet. Regional statistics 2014: sub-saharan Africa. 2015; http:// www.unaids.org/en/resources/campaigns/2014/2014gapreport/factsheet.

3. UNAIDS. Fast track-Ending the AIDS Epidemic by 2030. Geneva: Joint United Nations AIDS Programme; 2014.

4. WHO. HIV reporting: Global update on the health sector response to HIV, 2014. Geneva: World Health Organization; 2014.

5. Stats SA. Mid-year population estimates, South Africa 2015. Statistical release P 2015, 302. https://www.statssa.gov.za/publications/P0302/P03022015.pdf.

6. Shisana O, Rehle T, Simbayi L, Zuma K, Jooste S:South African national HIV prevalence, incidence and behaviour communication survey 2008: a turning tide among teenagers? 2009

7. Shisana O, Rehle T, Simbayi L, Zuma K, Jooste S. South African National HIV Prevalence, Incidence and Behaviour Survey, 2012. Cape Town: HSRC Press; 2014.

8. Statistics S: Mid-year population estimates 2010. 2010. [Online] https://www. statssa.gov.za/publications/P0302/P03022010.pd.

9. Segone M. Bridging the gap: The role of monitoring and evaluation in evidence-based policy making. Geneva: UNICEF, with DevInfo, IDEAS, MICS, World Bank; 2008.

10. UNAIDS W. Report on the global AIDS epidemic. Geneva: World Health Organization and UNAIDS; 2008.

11. GNP+, ICW, Young Positives, Engender Health, IPPF, UNAIDS. Advancing the Sexual and Reproductive Health and Human Rights of People Living With HIV: A Guidance Package. Amsterdam: The Global Network of People Living with HIV/AIDS (GNP+); 2009.
12. Englert Y, Van Vooren JP, Place I, Liesnard C, Laruelle C, Delbaere A. ART in HIV-infected couples: has the time come for a change of attitude? Hum Reprod (Oxford, England). 2001;16(7):1309-15.

13. Glynn JR, Buvé A, Caraël M, Kahindo M, Macauley IB, Musonda RM, et al. Decreased fertility among HIV-1-infected women attending antenatal clinics in three African cities. J Acquir Immune Defic Syndr. 1999;25(4):345-52.

14. Gilling-Smith C, Smith JR, Semprini AE. HIV and infertility: time to treat: There's no justification for denying treatment to patients who are HIV positive. BMJ. 2001;322(7286):566

15. Barreiro P, Duerr A, Beckerman K, Soriano V. Reproductive options for HIVdiscordant couples. AIDS Rev. 2006:8(3):158-70.

16. Bell E, Mthembu P, O'Sullivan S, Moody K. Sexual and Reproductive Health Services and HIV Testing: Perspectives and Experiences of Women and Men Living with HIV and AIDS. Reprod Health Matters. 2007;15(29 Supplement):113-35.

17. Beyeza-Kashesya J, Ekstrom AM, Kaharuza F, Mirembe F, Neema S, Kulane A. My partner wants a child: A cross-sectional study of the determinants of the desire for children among mutually disclosed sero-discordant couples receiving care in Uganda. BMC Public Health. 2010;10:247.

18. Abebe M, Endazenawb G. Fertility Intention and Family Planning use Among People Living with HIV/AIDS (PLHIV) on Follow up Care Western Shoa Zone, Anti-Retroviral Treatment (ART) Unit, Oromia, Ethiopia. http://asrjetsjournal.org/ index.php/American_Scientific_Journal/article/view/632/513.

19. Cooper D, Moodley J, Zweigenthal V, Bekker L-G, Shah I, Myer L: Fertility intentions and reproductive health care needs of People Living with HIV in Cape Town, South Africa: Implications for integrating reproductive health and HIV care services. AIDS Behav. 2009, DOI 10.1007/s10461-009-9550-1.

20. Gruskin S, Firestone R, MacCarthy S, Ferguson L. HIV and pregnancy intentions: Do services adequately respond to women's needs? Am J Public Health. 2008;98(10):1746-50.

21. Kaida A, Laher F, Strathdee SA, Janssen PA, Money D, Hogg RS, et al: Childbearing Intentions of HIV-Positive Women of Reproductive Age in Soweto, South Africa: The Influence of Expanding Access to HAART in an HIV Hyper-endemic Setting. Am J Public Health. 2009, Doi 10.2015/AJPH 2009.177469.

22. Rispel LC, Metcalf CA, Moody K, Cloete A, Caswell G. Sexual relations and childbearing decisions of HIV-discordant couples: an exploratory study in South Africa and Tanzania. Reprod Health Matters. 2011;19(37):184-93.

23. Tamene W, Fantahun M. Fertility desire and family-planning demand among HIV-positive women and men undergoing antiretroviral treatment in Addis Ababa, Ethiopia. Afr J AIDS Res. 2007:6:223-7.

24. Chen JL, Phillips KA, Kanouse DE, Collins RL, Miu A. Fertility desires and intentions of HIV-positive men and women. Fam Plan Perspect. 2001, check:144-165. https://www.guttmacher.org/journals/psrh/2001/07/fertilitydesires-and-intentions-hiv-positive-men-andwomen.

25. McClellan MK, Patel R, Kadzirange G, Chipato T, Katzenstein D: Fertility desires and condom use among HIV-positive women at an antiretroviral roll-out program in Zimbabwe: original research. Afr J Reprod Health. 2010;14(2):27-35

26. Ogilvie GS, Palepu A, Remple VP, Maan E, Heath K, MacDonald G, et al. Fertility intentions of women of reproductive age living with HIV in British Columbia, Canada. AIDS. 2007;21:S83-8.

27. Heard I, Sitta R, Lert F. Reproductive choice in men and women living with HIV: evidence from a large representative sample of outpatients attending French hospitals (ANRS-EN12-VESPA Study). AIDS. 2007;21:S77-82.

28. Mantell JE, Smit JA, Stein ZA. The right to choose parenthood among HIV-infected women and men. J Public Health Policy. 2009;30(4):367-78.

29. Finocchario-Kessler S, Bastos Fl, Malta M, Anderson J, Goggin K, Sweat MD, et al. Discussing childbearing with HIV-infected women of reproductive age in clinical care: a comparison of Brazil and the US. AIDS Behav. 2012;16(1):99-107.

30. Finocchario-Kessler S, Dariotis JK, Sweat MD, Trent ME, Keller JM, Hafeez Q, et al. Do HIV-infected women want to discuss reproductive plans with providers, and are those conversations occurring? AIDS Patient Care STDs. 2010;24(5):317-23.

31. Hayford SR, Agadjanian V. Providers' Views Concerning Family Planning Service Delivery to HIV-positive Women in Mozambique. Stud Fam Plan. 2010;41(4):291-300.

32. Rao A, Baral S, Phaswana-Mafuya N, Lambert A, Kose Z, Mcingana M, et al. Pregnancy Intentions and Safer Pregnancy Knowledge Among Female Sex Workers in Port Elizabeth, South Africa. http://journals.lww.com/ greenjournal/Abstract/2016/07000/Pregnancy_Intentions_and_Safer Pregnancy_Knowledge.4.aspx. 
33. Schaan MM, Taylor M, Puvimanasinghe J, Busang L, Keapoletswe K, Marlink R. Sexual and reproductive health needs of HIV-positive women in Botswana-a study of health care worker's views. AIDS Care. 2012;24(9):1120-5.

34. Schwartz SR, Mehta SH, Taha TE, Rees HV, Venter F, Black V. High pregnancy intentions and missed opportunities for patient-provider communication about fertility in a South African cohort of HIV-positive women on antiretroviral therapy. AIDS Behav. 2012;16(1):69-78.

35. Wagner GS, Linnemayr C, Kityo C, Mugyenyi P. Factors associated with intention to conceive and its communication to providers among HIV clients in Uganda. Matern Child Health J. 2012;16(2):510-8.

36. DOH. Negotiated Service Delivery Agreement. Pretoria: Department of Health (DOH); 2010.

37. DOH. National Health Insurance in South Africa: Policy paper. Government Notice: 657 of 12th August 2011, Gazette Number 34523. Pretoria: Department of Health (DOH). p. 2011.

38. GDOH. Gauteng department of health and social development annual performance plan 2011/12-2013/14. http://www.health.gpg.gov.za/ dohDocuments/Annual\%20Reports/ANNUAL\%20REPORT\%20\%20\%2020112012\%20Gauteng\%20Department\%20of\%20Health.pdf.

39. Stats SA. Census 2011 Gauteng Municipal report: Statistics South Africa, 2012. Report no.: 03-01-55 72 pp. http://www.statssa.gov.za/census/census_ 2011/census_products/GP_Municipal_Report.pdf.

40. DHP. Ekurhuleni health district; District health plan (DHP), 2012/13-13/14.

41. DHIS: Ekurhuleni health district; DHIS data 2013.

42. STATS SA. Employment, unemployment, skills and economic growth: An exploration of household survey evidence on skills development and unemployment between 1994 and 2014. https:/www.statssa.gov.za/presentation/ Stats\%20SA\%20presentation\%20on\%20skills\%20and\%20unemployment_ 16\%20September.pdf.

43. WHO. Global Health Sector Strategy on HIV/AIDS, 2011-2015. Geneva: World Health Organization; 2011.

44. Oladapo OT, Daniel OJ, Odusoga OL, Ayoola-Sotubo O. Fertility desires and intentions of HIV-positive patients at a suburban specialist center. J Natl Med Assoc. 2005;97(12):1672.

45. Smits AK, Goergen CA, Dalaney JA, Williamson C, Mundy LM, Fraser VJ. Contraceptive use and pregnancy decision making among women with HIV. AIDS Patient Care STDs. 1999;13(12):739-46.

46. Kirshenbaum SB, Hirky AE, Correale J, Goldstein RB, Johnson MO Rotheram-Borus MJ, et al. "Throwing the Dice": Pregnancy Decision-Making Among HIV-Positive Women in Four US Cities. Perspect Sex Reprod Health. 2004;36(3):106-13.

47. Nakayiwa S, Abang B, Packel L, Lifshay J, Purcell DW, King R, et al. Desire for children and pregnancy risk behavior among HIV-infected men and women in Uganda. AIDS Behav. 2006;10(1):95-104.

48. Dyer SJ, Abrahams N, Hoffman M, van der Spuy ZM. Men leave me as I cannot have children': women's experiences with involuntary childlessness. Hum Reprod. 2002;17(6):1663-8.

49. Dyer S, Abrahams N, Mokoena N, Van der Spuy Z. 'You are a man because you have children': experiences, reproductive health knowledge and treatment-seeking behaviour among men suffering from couple infertility in South Africa. Hum Reprod. 2004;19(4):960-7. Epub 2004 Mar 11.

50. Cooper D, Harries J, Myer L, Orner P, Bracken H. "Life is still going on": reproductive intentions among HIV-positive women and men in South Africa. Soc Sci Med. 2007;65(2):274-83.

51. Doyal $L$, Anderson J. 'My fear is to fall in love again...'How HIV-positive African women survive in London. Soc Sci Med. 2005;60(8):1729-38.

52. Paiva V, Ventura Filipe E, Santos N, Novaes Lima T, Segurado A. The right to love: the desire for parenthood among men living with HIV. Reprod Health Matters. 2003;11(22):91-100.

53. Sherr L, Barry N. Fatherhood and HIV-positive heterosexual men. HIV Med. 2004;5(4):258-63.

54. Chen JL, Phillips KA, Kanouse DE, Collins RL, Miu A. Fertility desires and intentions of HIV-positive men and women. Fam Plan Perspect. 2001:144-65.

55. Nóbrega AA, Oliveira FA, Galvão MT, Mota RS, Barbosa RM, Dourado I, et al. Desire for a child among women living with HIV/AIDS in northeast Brazil. AIDS Patient Care STDs. 21. https://www.ncbi.nlm.nih.gov/pubmed/ 17461721

56. Panozzoa L, Battegayb M, Friedlc A, Vernazzaa PL. High risk behaviour and fertility desires among heterosexual HIV-positive patients with a serodiscordant partner-two challenging issues. Women. 2003;46:60.
57. Englert Y, Van Vooren J-P, Place I, Liesnard C, Laruelle C, Delbaere A. ART in HIV-infected couples Has the time come for a change of attitude? Hum Reprod. 2001;16(7):1309-15.

58. Bharat S, Mahendra VS. Meeting the sexual and reproductive health needs of people living with HIV: challenges for health care providers. Reprod Health Matters. 2007;15(29):93-112.

59. Paiva V, Santos N, França-Junior I, Filipe E, Ayres JR, Segurado A. Desire to have children: gender and reproductive rights of men and women living with HIV: a challenge to health care in Brazil. AIDS Patient Care STDs. 2007;21(4):268-77.

60. SANAC: National Strategic Plan on HIV, STIs and TB, 2012-2016. South African National AIDS Council Pretoria; 2012.http://sanac.org.za/about-sanac/ the-national-strategic-plan-nsp-2012-2016-in-anutshell/.

\section{Submit your next manuscript to BioMed Central and we will help you at every step:}

- We accept pre-submission inquiries

- Our selector tool helps you to find the most relevant journal

- We provide round the clock customer support

- Convenient online submission

- Thorough peer review

- Inclusion in PubMed and all major indexing services

- Maximum visibility for your research

Submit your manuscript at www.biomedcentral.com/submit
) Biomed Central 